\title{
Um Modelo de Recomendação de Objetos Aprendizagem Baseado em Valores Culturais dos Estudantes
}

\author{
Felipe L. da Silva ${ }^{1}$, Sílvio C. Cazella ${ }^{12}$ \\ ${ }^{1}$ Programa de Pós-graduação em Informática na Educação - Universidade Federal do Rio \\ Grande do Sul (UFRGS) - Porto Alegre - RS - Brasil \\ ${ }^{2}$ Departamento de Ciências Exatas e Sociais Aplicadas - Universidade Federal de Ciências \\ da Saúde de Porto Alegre (UFSCPA) - Porto Alegre - RS - Brasil \\ fsilva@ufrgs.br, silvioceufcspa.edu.br
}

\begin{abstract}
Educational repositories provide access to learning objects designed by educators from different regions and cultures. However, when students use educational resources culturally incompatible to their values, they can experience conflicts in their learning process discouraging their engagement. Targeting this scenario, this paper presents an educational recommendation system model that is based on the cultural dimensions of learning framework to produce learning objects recommendations culturally adapted to student's profile.
\end{abstract}

Resumo. Os repositórios educacionais proporcionam oportunidade de acesso a objetos de aprendizagem elaborados por educadores de diferentes regiões e culturas. No entanto quando estudantes utilizam recursos educacionais não adaptados às suas características culturais, eles podem apresentar conflitos no seu processo de aprendizagem desestimulando o seu envolvimento. Nesse contexto, este artigo apresenta um modelo de sistema de recomendação educacional que se baseia nas dimensões culturais da aprendizagem para produzir recomendações de objetos culturalmente adaptados ao perfil educacional dos estudantes.

\section{Introdução}

No ensino superior, os estudantes que desejam aprimorar seus conhecimentos, muitas vezes, recorrem a internet para acessar materiais que os auxiliem nos seus estudos. Diante desse cenário, os objetos de aprendizagem (OA) são um dos tipos de recursos educacionais disponíveis. No Brasil, os OAs representam um dos principais recursos educacionais oferecidos no ensino a distância e semipresencial segundo o último Relatório Analítico da Aprendizagem a Distância no Brasil [Associação Brasileira de Educação a Distância, 2019]. Esses objetos normalmente contêm conteúdo instrucional ou oportunidades para a prática de tópicos de uma área de conhecimento e servem para apoiar os estudantes em suas necessidades de aprendizagem.

Os OAs são recursos tipicamente disponibilizados por meio de repositórios e podem ser elaborados e acessados por indivíduos de diferentes regiões e nações. Tal característica favorece o surgimento de ambientes digitais que hospedam itens criados a partir de contextos culturais diversos. 
O conteúdo multicultural dos repositórios traz desafios para estudantes que desejam utilizar OAs para complementar seus estudos. Isso, porque os educadores geralmente não estão cientes da existência de características culturais nas práticas educacionais [Roger et al, 2007]. Deste modo, práticas como a elaboração de OAs são alinhadas aos valores cultural do educador e, muitas vezes, não são culturalmente adaptadas aos estudantes. Por outro lado, o alinhamento cultural de práticas educacionais aos estudantes exerce papel importante no seu processo de aprendizagem. Divergências culturais entre a forma que o ensino é transmitido e os estudantes pode gerar conflitos no alcance de metas de aprendizado [Parrish e Linder-VanBerschout, 2010].

Devido à natureza inerentemente digital e a sua disponibilização por meio da internet, os OAs podem ser acessados por diferentes estudantes, inclusive, por aqueles para os quais não foram originalmente projetados. Tais características dificultam a elaboração de objetos culturalmente adaptados e uma alternativa é a recomendação de itens que atendam ao perfil cultural deles.

Dentre diferentes cenários de utilização, os sistemas de recomendações educacionais (SREs) são utilizados para tratar o problema da busca por objetos relevantes às configurações de aprendizados dos estudantes [Laisa et al, 2018]. Tais sistemas filtram OAs considerando as necessidades individuais deles, e ao mesmo tempo em que os auxiliam a lidar com a sobrecarga de informações, os orientam na obtenção de conhecimento e assim facilitam que alcancem seus objetivos educacionais. As diferentes propostas existentes, contudo, ignoram a inclusão de valores culturais em SREs [Laisa et al, 2018], mesmo que tal atributo seja considerado relevante na produção de recomendações educacionais [Maravanyika e Dlodlo, 2018].

Diante da necessidade de estudantes obterem itens adaptados ao seu contexto cultural, observa-se uma demanda por soluções tecnológicas que os apoiem. Deste modo, este artigo tem o objetivo de apresentar um modelo de recomendação de OAs que considera os valores culturais dos estudantes na produção de recomendações. Para tal, o modelo considera as dimensões culturais da aprendizagem propostas por Parrish e Linder-VanBerschout (2010). Tais dimensões descrevem oito características que podem ser manifestadas pelos estudantes em situações de ensino e aprendizagem e que revelam as diferenças culturais que influenciam no seu aprendizado.

Este artigo está organizado da seguinte forma: na Seção 2 são apresentados os trabalhos relacionados, na Seção 3 é apresentado o modelo de recomendação proposto e na Seção 4 o artigo é encerrado com as considerações finais e a previsão de trabalhos futuros.

\section{Trabalhos Relacionados}

Zangerle et al (2018) apresentam uma modelagem de perfil de usuário que incorpora as características acústicas de músicas que os usuários já ouviram assim como o contexto cultural provenientes da sua localização para a produção de recomendações musicais. Esse modelo considera as dimensões culturais de Hofstede nas recomendações. Zangerle et al (2020) expandem seu trabalho inicial e apresentam detalhes do processo de recomendação e dos experimentos realizados. Gao et al (2018) propõem um algoritmo de filtragem colaborativa baseada em itens que inclui características culturais dos usuários na produção de seus resultados. 
Apesar desses trabalhos apresentarem propostas relacionadas a inclusão de dimensões culturais em recomendações eles apresentam limitação quanto ao escopo de aplicação. Ambos não são projetados para o domínio da educação e, portanto, não consideram características culturais que contribuam para o aprendizado do usuário.

Savard et al (2020) elabora um modelo conceitual de cultura e identifica variáveis culturais nas práticas pedagógicas. A partir disso propõem um sistema orientador baseado em conhecimentos que auxilia educadores a elaborar práticas adaptadas a uma cultura diferente da sua. Embora aplicada ao domínio da educação, a proposta não se aplica a recomendação de OAs. Além disso, seu escopo incluí orientar educadores na elaboração das práticas instrucionais e não na recomendação para estudantes.

\section{Modelo de Recomendação de Objetos de Aprendizagem}

Esta seção descreve o modelo proposto. Por meio dele objetiva-se incorporar a percepção dos valores culturais dos estudantes em um SRE e produzir recomendações de OAs culturalmente adaptados a eles. Para esse objetivo, o modelo considera as dimensões culturais da aprendizagem, do inglês Cultural Dimensions of Learning Framework (CDLF), de Parrish e Linder-VanBerschout (2010).

A CDLF apresenta oito dimensões que correspondem aos valores culturais manifestadas por estudantes durante o processo de aprendizagem. Essas dimensões são divididas em três categorias: relações sociais, crenças epistemológicas e percepções temporais. A categoria de relações sociais possui as dimensões de igualdade ou autoridade, individualismo ou coletivismo, criação ou desafio; a categoria de crenças epistemológicas possui as dimensões de busca de estabilidade ou aceitação da incerteza, argumentação lógica ou ser razoável, foco em causalidade ou foco em sistemas complexos; e a categoria de percepções temporais possui as dimensões de foco no horário ou foco no evento e tempo linear ou tempo cíclico.

Além de definir as dimensões, a CDLF define a série de sua variabilidade conforme podem ser manifestadas em situação de aprendizado. Parrish e LinderVanBerschout (2010) apresentam um questionário como instrumento de medição do nível de concordância dos estudantes em relação às dimensões culturais. Nesse instrumento, cada dimensão é representada por uma linha contínua que possui dois valores culturais opostos em suas extremidades (ex. individualismo e coletivismo). Os estudantes indicam seu nível de concordância selecionando um valor em escala de $1 \mathrm{a}$ 10. Valores menores indicam que o estudante tende a característica da esquerda da dimensão cultural enquanto valores maiores indicam que ele tende a característica da direita.

\subsection{Componentes e Interações}

O modelo é apresentado na Figura 1. Ele apresenta quatro componentes principais que interagem entre si: os educadores, os estudantes, o repositório educacional e o SRE consciente de valores culturais. Os educadores são os usuários que elaboram OAs e os inserem no repositório juntamente com outras informações relacionadas eles, os estudantes são aqueles que acessam os OAs para obter conteúdo instrucional ou realizar uma prática educacional, o repositório é a plataforma digital que hospeda os OAs e as informações associadas a eles, e o SRE é um componente do repositório que mantém 
IX Congresso Brasileiro de Informática na Educação (CBIE 2020)

Anais Estendidos do XXXI Simpósio Brasileiro de Informática na Educação (SBIE 2020)

um perfil de cada estudante e que processa essas informações a fim produzir uma lista com recomendações de OAs.

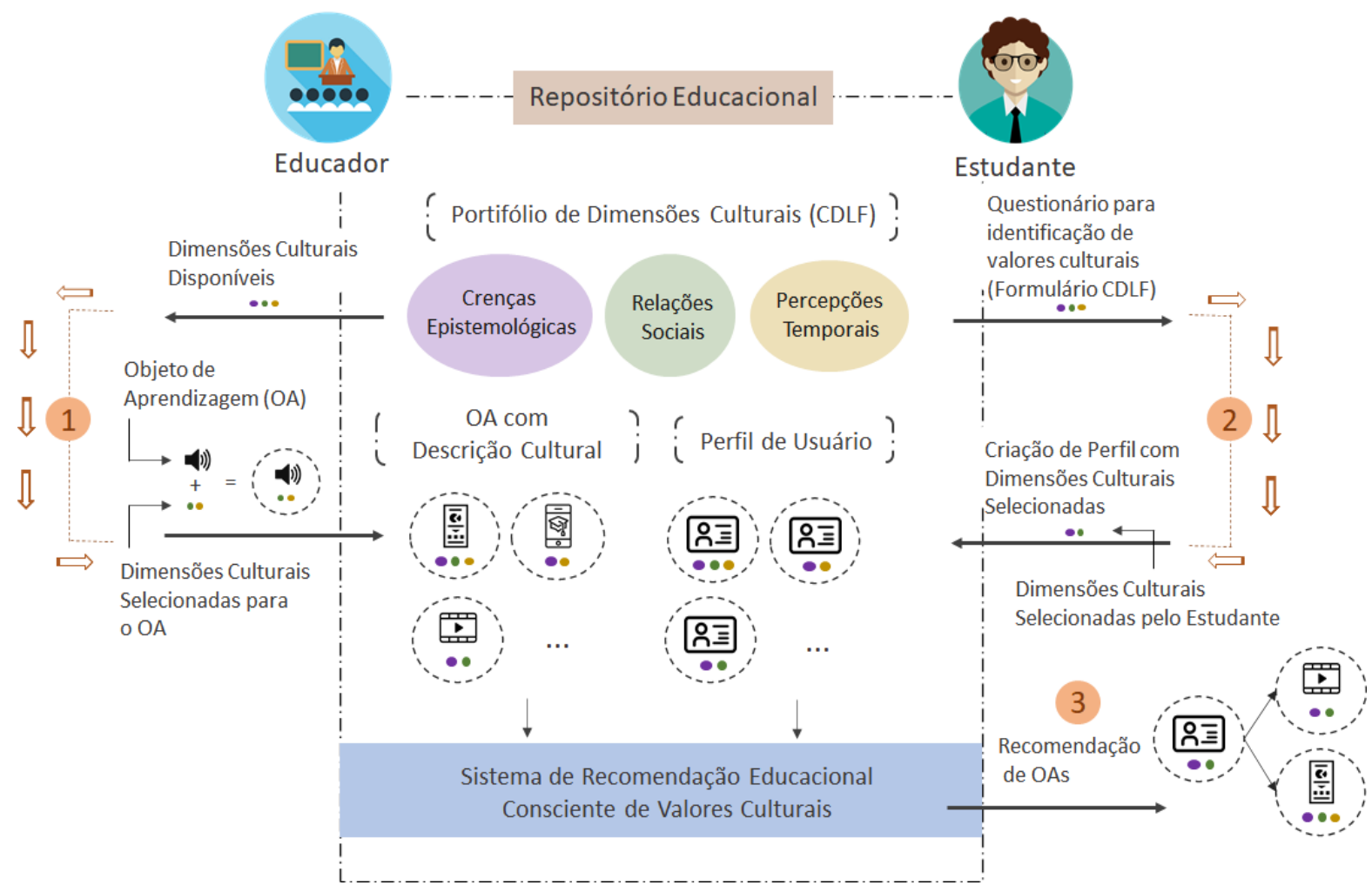

Figura 1. Modelo de Recomendação de OAs Baseado em Valores Culturais

A primeira interação do modelo é a inserção de um OA no repositório. Após elaborar um objeto, os educadores devem registrá-lo na plataforma juntamente com as suas respectivas informações culturais. Tais informações são os valores culturais relacionados ao OA. O repositório mantém um portfólio com os possíveis valores. Eles correspondem às dimensões culturais que compõe a CDLF.

Além das informações culturais dos OAs, o repositório também gerencia as informações culturais dos estudantes (interação 2). Isso ocorre de forma explícita através da captura das dimensões culturais deles por meio do questionário da CDLF. Essa etapa é essencial para o modelo, visto que essas informações são fundamentais para a criação dos perfis dos usuários. Esse perfil incorpora as preferências dos estudantes e as suas dimensões culturais de aprendizagem.

Depois que as informações da $1^{a}$ interação e da $2^{a}$ interação estão registradas, o SRE utiliza o perfil de usuário e as características culturais associadas a um OA para produzir as recomendações (interação 3). Essas entradas são consumidas por mecanismos de filtragem colaborativa e baseada em conteúdos adaptados. Os objetos identificados como de interesse dos estudantes (ex. aqueles que outros usuários com interesses parecidos classificaram como relevantes para si) e que estão associados a dimensões culturais relacionadas às dimensões registradas no perfil do usuário são recomendados. Novos OAs são recomendados com base nas suas dimensões culturais. 


\section{Considerações Finais}

Neste artigo foi apresentado um modelo que inclui as características culturais dos estudantes na produção de recomendações de OAs. Tal modelo é parte introdutória de um projeto de pesquisa de desenvolvimento de um SRE consciente de características culturais dos estudantes. Por meio dele espera-se contribuir apresentando direcionamentos sobre como considerar tais características em recomendações educacionais. Além disso, é apresentado uma estratégia para inclusão de uma nova funcionalidade em SREs. Essa estratégia objetiva tornar as recomendações mais adequadas às necessidades de aprendizagem dos estudantes ao considerar seus valores culturais. Como trabalho futuro prevê-se a implementação de um SRE que incorpore o modelo proposto assim como a realização de experimentos para validar seus impactos no processo de aprendizagem dos estudantes.

\section{Referências}

Associação Brasileira de Educação a Distância. (2019) "Censo EAD.BR 2018: Relatório analítico da aprendizagem a distância no Brasil", http://abed.org.br/arquivos/CENSO_DIGITAL_EAD_2018_PORTUGUES.pdf, Junho.

Gao, Z., Liu, Y., Xiao, K., Yang, Y. (2018) "Cultural Distance Awared Collaborative Filtering Algorithm in Recommendation System". IOP Conference Series Materials Science and Engineering.

Laisa, J., Medeiros, T., Aranha E., Silva, T. (2018) "Uma Revisão Sistemática da Literatura sobre Sistemas de Recomendação Educacional”. Anais do IX Computer on the Beach, Florianópolis, p. 751-760

Maravanyika, M.; Dlodlo, N. (2018) "Selecting Attributes for Inclusion in an Educational Recommender System Using the Multi-attribute Utility Theory". Applied Informatics: International Conference on Applied Informatics 2018,

Parrish, P., Linder-VanBerschout, J. (2010) "Cultural Dimensions of Learning: Addressing the Challenges of Multicultural Instruction.". International Review of Research in Open and Distance Learning, p. 1-19.

Rogers, P., Graham, C., Mayes, C. (2007) "Cultural competence and instructional design: Exploration research into the delivery of online instruction crossculturally". Educational Technology Research \& Development, p. 197-217.

Savard, I., Bourdeau, J., Paquette, G. (2020) "Considering cultural variables in the instructional design process: A knowledge-based advisor system". Computers \& Education.

Zangerle, E., Pichl, M., Schedl, M. (2018) "Culture-Aware Music Recommendation". Proceedings of the 26th Conference on User Modeling, Adaptation and Personalization, Singapura, P. 357-358.

Zangerle, E., Pichl, M. and Schedl, M. (2020) "User Models for Culture-Aware Music Recommendation: Fusing Acoustic and Cultural Cues". Transactions of the International Society for Music Information Retrieval, p.1-16. 